\section{Nach der Krebsdiagnose häufen sich Herztod und Suizide}

\author{
Wenn Patienten die Diagnose „Krebs“ erfahren, verdüstert sich ihre \\ Prognose: nicht nur durch die maligne Erkrankung selbst, sondern auch \\ durch einen Anstieg kardiovaskulärer Todesfälle und Suizide.
}

$\mathrm{D}^{\mathrm{i}}$ ie sehr zuverlässigen nationalen Krebs- und Sterberegister in Schweden verzeichnen zwischen 1991 und 2006 erstmalige Krebsdiagnosen bei 534.154 Patienten. Während der Nachbeobachtung wurden 786 Suizide registriert. Im Vergleich zu Patienten ohne Krebsdiagnose errechnet sich im Langzeitverlauf eine Suizidrate von 0,36 pro 1.000 Personenjahre, die doppelt so hoch liegt wie bei Patienten ohne eine solche Diagnose $(0,18)$. Das Risiko war in der ersten Woche nach der Diagnose besonders stark, nämlich um das 12,6-Fache erhöht. Während der folgenden zwölf Wochen erreichte es immer noch das 4,8-Fache, bei den prognostisch ungünstigen Malignomen von Ösophagus, Leber und Pankreas sogar das 16-Fache.
Ähnlich verhält es sich bei kardiovaskulären Todesfällen, die bei Krebspatienten im Gesamtverlauf dreimal häufiger auftreten (23,10 vs. 7,53 pro 1.000 Personenjahre). Auch hier war das Risiko in der ersten Woche nach Diagnose am stärksten (um das 5,6-Fache) erhöht. Prof. Heinrich Holzgreve

Fang $\mathrm{F}$ et al. Suicide and cardiovascular death after a cancer diagnosis. New Engl J Med. 2012;366(14):1310-8.

Kommentar von Prof. Holzgreve: Jeder Patient, der seine Diagnose „Krebs" erfährt, muss damit rechnen, dass seine Lebensprognose durch diese Krankheit - möglicherweise entscheidend - beeinträchtigt wird. Die Ergebnisse dieser Analyse dokumentie- ren eindrucksvoll, wie sehr nicht nur der physische Aspekt der Erkrankung, sondern auch die psychischen Reaktionen nach der Diagnosemitteilung lebensbegrenzend sind.

„Auch die psychischen Reaktionen nach der Diagnose sind lebensbegrenzend."

Die eminente psychische Traumatisierung ist daran erkennbar, dass sowohl kardiovaskuläre Todesfälle als auch die Suizidrate zwar während der ganzen Beobachtungsdauer erhöht bleiben, doch in den ersten Wochen und bei prognostisch ungünstigen Karzinomen besonders hoch liegen. Jeder Patient mit einer Krebsdiagnose ist also auch bedroht durch einen fatalen Ausgang, der durch Operation, Strahlen- und Chemotherapie nicht gemildert wird.

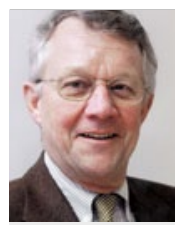

Prof. Heinrich Holzgreve Internist, Kardiologische Praxis, München h.holzgreve@t-online.de

\title{
Missverständnisse vorprogrammiert
}

Zum Zeitpunkt der Krebsdiagnose kennen die Patienten viele onkologische Begriffe noch nicht. Nachfragen kann helfen, das Verständnis in den ersten Arzt-Patienten-Gesprächen zu verbessern.

$\mathrm{D}$ as hat eine Studie aus Holland hervorgebracht, in der anhand bereits untersuchter Beispiele typischer onkologischer Aufklärungssituationen aus britischen Studien und an die holländischen Bedingungen angepasste Situationen mit Laien untersucht wurden. 149 Teilnehmer sollten sich intensiv die Situation einer Krebsdiagnose vorstellen. Dann erhielten sie insgesamt 10 Beschreibungen von Szenarien eines Arzt-Patienten-Gesprächs. Danach füllten sie Fragebögen aus, die das Verstehen bestimmter Fachtermini, die Sicherheit bezüglich des richtigen Verständnisses und die Sorge, etwas nicht richtig verstanden zu haben, abfragten. Außerdem wurde anhand von weiteren kurzen Szenen abgefragt, ob die dargestellte Kommunikation wirksam war, ob sie in die Lage versetzte, Entscheidungen mit zu treffen und ob sie Vertrauen schuf.
In den Szenen verwendete der Arzt einmal viele Fachtermini, ein anderes Mal bediente er sich der Alltagssprache.

Im Mittel verstanden die Teilnehmer zwar bei der Mehrheit der Fachtermini, ob sie etwas Gutes oder etwas Schlechtes bedeuteten (6,8 von 10$)$, aber nur 2,2\% verstanden alle Begriffe. So wurde beispielsweise besonders oft der Begriff „Remission" negativ verstanden. Bei denjenigen Teilnehmern, die viele Begriffe korrekt als günstig oder schlecht eingeordnet hatten, stellten die Untersucher auch eine größere Sicherheit bezüglich des Verständnisses dar. Auch gaben sie Sorgen und Ängste eher den vorgestellten Szenarien entsprechend an, während diejenigen, die wenige Begriffe richtig eingeordnet hatten, auch eher unsicher waren, ob sie alles richtig verstanden hatten. Auf die vorgestellten Szenarien äußerten sie häu- figer unangemessene Sorgen. Ob Fachoder Alltagssprache verwendet wurden, entschied wenig, ob die Gespräche als effektiv und das Vertrauen fördernd empfunden wurden. Die eigene Zuversicht bezüglich des Verständnisses im Gespräch war aber signifikant mit der Einschätzung der Effektivität des Gesprächs assoziiert $(\mathrm{p}<0,01)$.

Fazit: Laien, also auch Patienten mit einem neu diagnostizierten Tumor, verstehen gebräuchliche Fachtermini in der Onkologie häufig nicht richtig. Zudem beeinflusst das Vertrauen in das eigene Verständnis stark die Effektivität der Arzt-Patienten-Kommunikation. Es ist wichtig, sich des richtigen Verständnisses im Gespräch durch Nachfragen zu versichern, um unnötige Ängste zu vermeiden und eine Basis für die Beteiligung der Patienten an wichtigen Entscheidungen aufzubauen. Friederike Klein

Pieterse $\mathrm{AH}$ et al. Lay understanding of common medical terminology in oncology. Psychooncology. 2012 May 10. doi: 10.1002/ pon.3096. [Epub ahead of print]. 